\title{
LA LÓGICA DE LA JUSTICIA EN UNA COMUNIDAD INDÍGENA MIXE ${ }^{1}$
}

\begin{abstract}
Elisa Cruz Rueda ${ }^{2}$
elisacruzrueda@hotmail.com

LGAI

Universidad Autónoma de Chiapas

RESUMEN

México

Se muestran casos relevantes atendidos por las autoridades de la comunidad de estudio, algunos presenciados y otros reconstruidos a través de narraciones y algunos documentos. Con esto se hace una reflexión sobre los criterios para su resolución, la diferencia entre asuntos graves y leves, así como las normas orales y escritas, su naturaleza y diferencias, mostrando la especificidad del Derecho que se construye en la comunidad indígena, que finalmente se encuentra inmersa en un sistema más amplio que es el del Estado mexicano. Lo que se presenta es una mínima parte de la investigación de campo y documental — histórica, social, teórico y conceptual—, dejando de lado los debates desarrollados en el seno de la Antropología jurídica y la Sociología del derecho sobre pluralismo jurídico, interlegalidad, sistemas normativos indígenas y la relación de los pueblos indígenas y el Estado (cfr. Santos 1991, 1995, 1998; Sierra 1987, 2004a y b, 2006; Valdivia 2001, 2010, así como muchos otros autores).
\end{abstract}

Palabras clave: Derecho, derecho indígena, normas jurídicas orales y escritas. 


\section{SUMMARY}

Relevant cases addressed by the authorities of the study community are presented, some of them witnessed first-hand and others reconstructed through narrations and a few documents. A parallel reflection is developed on the criteria for their resolution, the difference between grave and minor matters, and oral and written norms and their nature and differences, demonstrating the specificity of the law that is constructed in the indigenous community, which in the end finds itself immersed in a broader system, that of the Mexican State. A minimal part of the field research and documentation (historic, social, theoretical, and conceptual) is presented, leaving aside debates developed in the core of juridical anthropology and sociology of law on juridical pluralism, inter-legality, indigenous normative systems, and the relation of indigenous peoples and the State (Cfr. Santos 1991, 1995 and 1998; Sierra 1987, 2004a and b, 2006 and Valdivia 2001 and 2006, and several other authors).

Key words: Law, indigenous law, oral and written juridical norms. 


\section{INTRODUCCIÓN}

Para comprender el planteamiento central sobre La lógica de la Justicia en una comunidad indígena mixe, considero necesario precisar algunos datos de contexto. El estudio se realizó en San Marcos Móctum, del estado de Oaxaca, que pertenece al grupo etnolingüístico mixe, la mayor parte de la población de este grupo se concentra en el único Distrito Judicial en su tipo en la República mexicana que lleva el nombre de un pueblo indígena, es decir, el Distrito Mixe. ${ }^{3}$

La comunidad de referencia es la comunidad-agencia de San Marcos Móctum -en adelante Móctum-, esta doble característica se debe a que jurídicamente y desde el punto de vista del Derecho agrario, su forma de tenencia de la tierra es comunal, y desde el derecho municipal como agencia de policía forma parte de la estructura municipal de Totontepec, poblado que es de igual forma comunidad-cabecera. Entre estos dos poblados existe una historia de tensión que se remonta hasta mucho antes de la Colonia, según la memoria colectiva (Gómez Bravo 2004). Historia que comparten, de una u otra manera, con las otras nueve comunidades-agencias que conforman el municipio, pero a diferencia de éstas Móctum se ha colocado con mayor fuerza y presencia frente a la cabecera, confrontando los intereses caciquiles de sus elites, logrando mejores resultados, por ejemplo, el respeto a sus límites territoriales.

Un concepto clave para este estudio es el de campo jurídico, el cual defino como el espacio, no precisamente físico, en el que se relacionan distintos derechos, como es el indígena y el del Estado, no es uniforme en tanto los sistemas jurídicos que lo conforman se relacionan entre sí y están articulados bajo la égida del Derecho estatal, que constituye el sistema jurídico hegemónico, que históricamente se ha impuesto a otros ordenamientos jurídicos como los sistemas jurídicos indígenas. El campo jurídico en la región de estudio comprende distintos niveles legales y políticos: la Federación, el estado de Oaxaca, la cabecera del municipio de Totontepec Villa de Morelos y sus agencias, en particular la agencia de Móctum. La circunscripción territorial del campo jurídico en el caso de estudio se ubica — sin considerar el nivel de la Federación — en una zona indígena, en la 
que es posible ver la imbricación de sistemas legales y la hegemonía del Estado y cómo este influye en las trasformaciones del Derecho y las dinámicas del poder. ${ }^{4}$ (Ver mapa.)

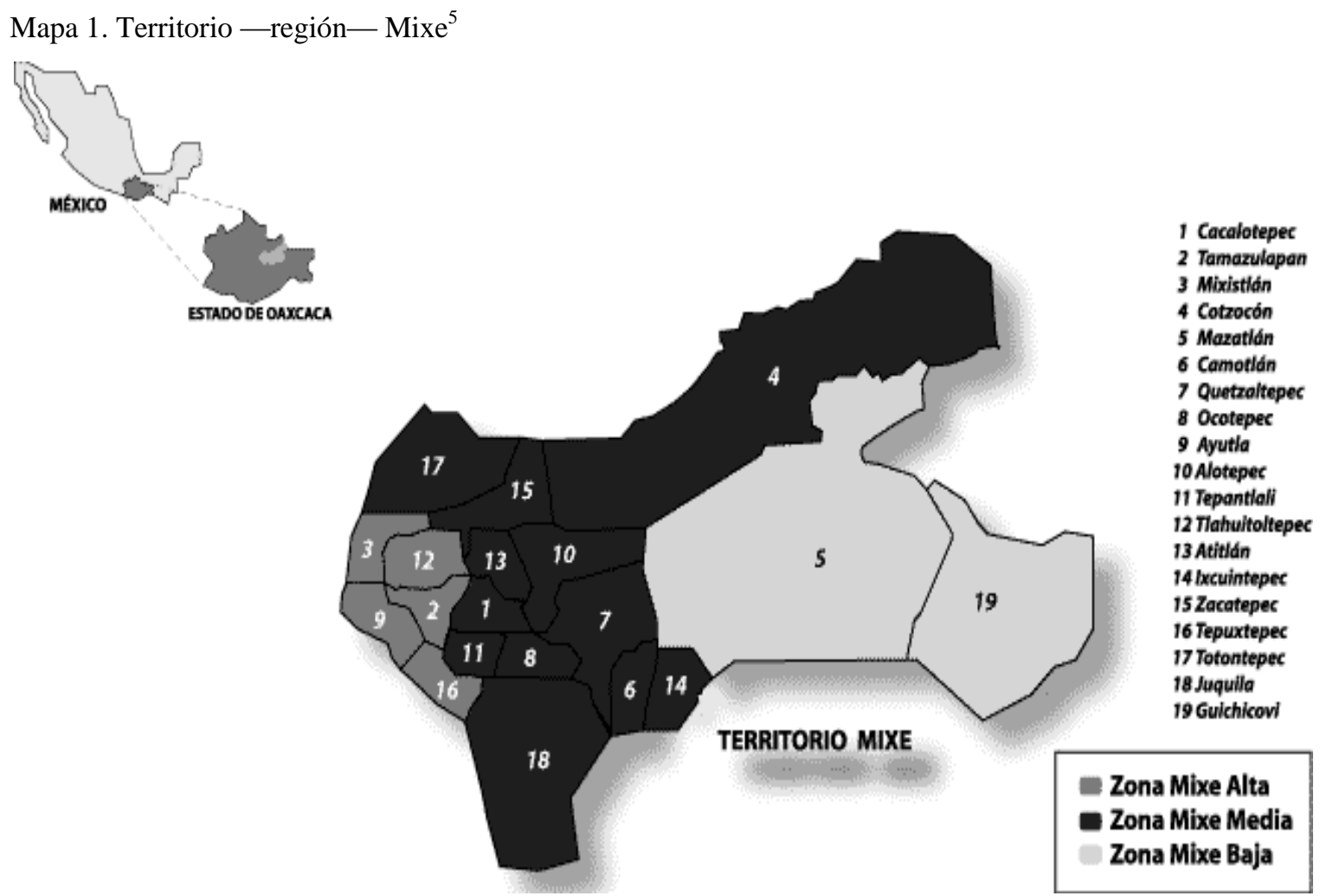

Fuente http://www.redindigena.net/ser/pueblomixe/localizacion.html

\section{EL DERECHO Y SU CONSTRUCCIÓN DESDE LAS DINÁMICAS SOCIALES DE MÓCTUM}

Para abordar el tema que nos ocupa, me centro en el estudio del Derecho en el ámbito local de Móctum, recurriendo a dos perspectivas de análisis dentro de la Antropología jurídica: la procesual y la normativa. Desde la primera perspectiva se considera al derecho como producto de la dinámica social, los usos del poder, la cultura y la historia de un pueblo; desde la segunda, interesa el precepto legal en sí mismo, sin considerar otros aspectos. En la conjunción de ambas perspectivas me propuse reconstruir las normas que regulan las dinámicas de la comunidad y de sus miembros concretamente en el sistema de cargos, esto a partir de la observación de las relaciones sociales, del ejercicio de los cargos y de la resolución de disputas, para dilucidar la génesis —origen, creación y fuentes - de las normas y el sentido en que son usadas. Para contextualizar y 
entender estos criterios y normas - la mayoría orales pero otras escritas en un Reglamento Interno promovido por lo que era el promotor agrario - adscrito a la Secretaría de la Reforma Agraria antes de las reformas al artículo 27 constitucional de 1992 - refiero los factores y circunstancias de los casos que dieron lugar a las normas y que indudablemente están implicados en la lógica de «hacer justicia». De esta manera, se hace una distinción — a nivel de la comunidad como nivel legal— entre las normas orales y las normas escritas; las primeras son acuerdos tomados por la mayoría de comuneros y ciudadanos en asamblea, a las que se suele hacer referencia como «el reglamento», que para evitar confusiones agregaremos que es oral. Estas normas las reconstruí a partir de las narraciones de casos que las autoridades pasadas y actuales resolvieron y se presentan aquí para facilitar su análisis — más que para formular un código o reglamento escrito-; en esta redacción de las normas orales se trató de respetar la narrativa de las personas. El segundo tipo de normas se presentan según como fueron escritas en el estatuto de la comunidad o Reglamento Interno, que como se ha dicho fue elaborado entre la asamblea de comuneros y un promotor de la Secretaría de la Reforma Agraria.

Como se mencionó, entre Móctum y Totontepec como cabecera del municipio existen tensiones, una de ellas la invasión de terrenos por algunas personas de este poblado en contra de aquél. Indudablemente el conflicto influyó en sus dinámicas internas e intercomunitarias, lo cual se expresa en el Derecho vigente en ambas comunidades.

Para comprender cómo se conforma el Derecho y cuál es la lógica de la justicia o de «hacer justicia» en Móctum, se estudian y muestran las normas contextualizándolas en la realidad de la comunidad y explicándolas según los casos que les dieron origen. El análisis se presenta de esta manera porque las normas abordadas únicamente como preceptos de conducta deseada o como sanciones posibles — sean escritas u oralesexpresan aspectos formales del Derecho en Móctum y, de alguna manera, muestran parcialmente una realidad. Por ello la norma, como traducción de la conducta humana a un «deber ser»y su mera trascripción o enunciación, tiene limitaciones prácticas para exponer las dinámicas del poder y los usos del Derecho en la comunidad. 
En este sentido soy conciente de las implicaciones en la metodología que apliqué sobre la reconstrucción de las normas orales, dados los riesgos de plasmar la norma oral a la manera de la norma escrita. Por un lado, la norma escrita tiene cierta garantía de no ser alterada tan fácilmente, y por lo mismo es difícil su manipulación cuando se involucran relaciones y usos de poder por parte de usuarios y autoridades. Por otro, la desventaja que presenta la norma escrita es que puede prestarse a imponer como hechos dados e inamovibles lo que se escribe. Sin embargo, las comunidades indígenas se han apropiado de la norma escrita incorporándola a su sistema jurídico interno, como una herramienta de invocación y ejercicio de sus derechos de redactar normas para regular por sí y para sí sus dinámicas locales, sin dejar de lado la norma oral.

Por su parte, las ventajas de la norma oral están marcadas por las desventajas de la norma escrita, es decir, a diferencia de ésta es flexible pues puede incorporar con mayor facilidad los hechos prácticos de la vida cotidiana, por lo que facilita la resolución de disputas. Sin embargo, partiendo de los criterios que el Derecho mexicano tiene para la modificación e interpretación de una norma escrita, en el caso de la norma oral los márgenes de interpretación son más amplios, lo que puede prestarse a manipulación en la aplicación de una norma a un caso concreto, más cuando intervienen relaciones y usos de poder, lo que también se da en el caso de la norma escrita, sin embargo, da lugar a menos ambigüedades porque no depende de la memoria colectiva sino de lo que está por escrito.

En el caso que se estudia, son tres aspectos a destacar de las normas orales: 1) Son normas no escritas que la comunidad se ha dado, se elaboran y actualizan a través del tiempo, la mayoría a partir de casos concretos que son resueltos en asamblea o por las autoridades en turno, tanto agrarias como de la agencia municipal. 2) Son normas que resultan de la atención a los asuntos que presentan los miembros de la comunidad en las oficinas de la agencia de policía de Móctum. 3) Resultan de la atención a diversos casos que revelan la emergencia de nuevos sujetos — como las mujeres - en las dinámicas internas cotidianas. ${ }^{6}$ En la observación de casos, puede dar cuenta de la influencia del 
Derecho positivo, sobre todo en el discurso y en el lenguaje de los usuarios y autoridades que invocan derechos ya sean constitucionales, indígenas, humanos o de las mujeres.

Esas normas —escritas o no- son el resultado de la atención y resolución de los conflictos y disputas, hacen referencia a los asuntos relevantes que más importan a la comunidad por estar íntimamente relacionadas con su historia y cultura. Es decir, son asuntos que muestran las conductas que se consideran obligatorias, permitidas y prohibidas en y por la comunidad, también expresan los derechos reconocidos y las obligaciones impuestas, por ejemplo, el tequio o el cargo, los criterios de adscripción, a quiénes se considera o se pueden considerar como comuneros, o quiénes pueden llegar a serlo. El derecho en la comunidad está constituido de estas normas que resultan determinadas por sus formas de organización social, su identidad cultural $^{7}$ y por su relación con los otros niveles del campo jurídico. Por ello, estas normas son construidas y trasformadas en las propias dinámicas sociales, históricas y culturales de la comunidad.

El interés central es mostrar la dinámica del derecho en Móctum, a través de la puesta en marcha de la estructura comunitaria para atender los asuntos que muestran a su vez la oposición de intereses en temas que son considerados por los pobladores como de especial importancia, y que por ello son considerados conflictos relevantes (Krotz 1995).

Para comprender la especificidad del Derecho en la comunidad es importante recordar que no se puede hablar de «un derecho mixe», pero sí de principios generales de ese derecho que tienen su expresión y aplicación particular en cada comunidad, pues los grupos etnolingüísticos no son homogéneos y uniformes, por el contrario manifiestan diversidad interna. En el caso de los mixes, esta diversidad se encuentra dada por los procesos históricos diferenciados de cada comunidad, en los que han influido aspectos como el ecológico, el demográfico, el geográfico, el económico y el social. Por esta circunstancia, no se puede hablar de un «ser mixe» sino de distintas maneras de ser mixe, lo que implica entender al sistema jurídico en la comunidad como parte de una realidad histórica y cultural compartida con el grupo etnolingüístico al que pertenece, pero con 
determinadas características, manifestaciones y diversidades concretas. Estas determinan la construcción y trasformación del Derecho en la comunidad indígena de que se trate y, a la vez, su realidad y las manifestaciones de la misma son influenciadas y determinadas por el mismo Derecho.

\section{LA LÓGICA JURÍDICO-CULTURAL EN LA COMUNIDAD}

En el caso de Móctum se puede observar que los conflictos están relacionados principalmente con cuatro aspectos de la vida de la comunidad: 1) los cargos y servicios, 2) el uso y tenencia de la tierra, 3) los recursos económicos o apoyos que vienen del municipio o del gobierno estatal, y 4) el papel de la mujer en la vida comunitaria.

Los asuntos son relevantes cuando evidencian o muestran el interés de la comunidad, los usos del poder y los procedimientos de resolución de sus conflictos. El control de estos asuntos relevantes y otros procesos comunitarios permite que exista una menor injerencia del exterior por parte de los agentes del Estado, frente a los cuales la ideología armónica (cfr. Nader 1998) ${ }^{8}$ ayuda a crear una idea de solidaridad y consenso al interior, que sale a relucir en los asuntos cuando la autoridad señala que las decisiones que se toman son por mayoría y acuerdo de todos (cfr. Cruz 2004, el caso de la disputa entre mujeres). De igual forma, podemos decir que son asuntos base de los que se derivan otros asuntos y conflictos.

De esta manera, a partir de las narraciones de los habitantes sobre las normas que se han dado, llegué a la conclusión de que los asuntos relevantes y base en Móctum son los que conciernen a:

1) Los cargos y servicios a la comunidad, el uso de la tierra y cumplimiento de las obligaciones asignadas por la asamblea, esto es una expresión de la voluntad de continuar siendo parte de la comunidad.

2) El ajuste de la conducta individual a lo que socialmente se espera y que afecta la vida y quehacer de las personas. ${ }^{9}$ Por ejemplo, solicitud y ejercicio de derechos de membresía como comunero o ciudadano, uso de la tierra, propiedad individual 
sobre los productos de la siembra o la cosecha. También intervienen las autoridades comunitarias, cuando hay riñas y lesiones graves que dejan cicatriz o dejan incapaz a una persona para realizar su trabajo cotidiano, o bien, en caso de chismes y celos. En ninguno de los casos se buscó por parte de los involucrados el arreglo privado o entre ellos, más bien se acudió a la autoridad; tampoco se dieron casos de denuncia contra los hombres por chisme.

3) Daños al patrimonio de una persona o familia, como daños en la milpa, robos en general, pero sobre todo de gallinas o frutos — café, plátano, limón-, o incumplimiento del pago de daños o de un trato o acuerdo. Todos estos tienen reparación económica o en especie si no se pone en riesgo la vida o la integridad de las personas - homicidio o lesiones graves que pongan en riesgo la vida-, o bien si no se hace uso de armas blancas o de fuego, o uso de la violencia física. No se presentó ninguno de estos casos durante mi estancia. No se reportan homicidios pero sí decesos por enfermedad o accidente, en este caso se da parte al síndico de Totontepec y éste a su vez al Ministerio Público que se encuentra en el Distrito, en Zacatepec.

4) Vigilancia sobre la autoridad en cuanto al manejo de recursos o apoyos económicos canalizados a través de programas gubernamentales. Se trata en asamblea o el agente manda a traer a la persona que está cuestionando su gestión, para «meterlo» en la cárcel o multarlo, si no tiene pruebas - testigos.

5) Acatamiento de la voluntad de la asamblea, relacionado sobre todo con el uso de la tierra y la inclusión o expulsión de un comunero. Por ejemplo, sucede cuando una mujer se casa con hombre fuereño —antes de 1990 que no se reconocían derechos a las mujeres-, cuando una persona nacida en Móctum ha salido de la comunidad para trabajar o estudiar y no coopera, y después quiere regresar y exige respeto a sus derechos de comunero.

En total se pudieron documentar veinte asuntos —además de los documentados en Totontepec- (para ambos casos, cfr. Cruz 2007), de los cuales por cuestión de espacio sólo se exponen cuatro casos, criterios generales y un panorama general del tipo de 
asuntos que las personas en Móctum disputan, que incluye una variedad de conductas como la siembra, el pastoreo, construcción de casas, ranchos, daños sobre éstos, ofensas entre las personas —riñas, lesiones, agresiones verbales, chismes, difamación-, cuestionamiento de la autoridad, alteración del orden después de las 10 pm, ${ }^{10}$ trasgresión al acuerdo colectivo o de asamblea, problemas intrafamiliares - abandono de hijos, como el cuidado de los niños es asumido por los abuelos o familiares la autoridad sólo interviene en caso de que se lo pidan, infidelidad, golpes a la esposa-. Los cuatro asuntos que se abordarán a continuación versan sobre: 1. Daños en parcela 2. Robo, 3. Uso y propiedad de la tierra, y 4. Derechos agrarios y de pertenencia de la mujer.

\section{ASUNTOS RELEVANTES SOBRE EL PATRIMONIO PARTICULAR: ROBOS Y DAÑOS}

En el marco comunalista y bajo el principio de la comunalidad ${ }^{11}$ se reconocen derechos individuales. Como parte de la norma interna se hace valer el derecho que tiene una persona sobre cosas o animales; a los comuneros se les reconoce el derecho que tienen sobre su casa, su solar urbano y su parcela o los frutos que cosechan, siempre y cuando cumplan con sus obligaciones y servicios para la comunidad. Frente a la propiedad individual existe la propiedad colectiva sobre la tierra, es decir, es de todos y de nadie en lo individual, sin embargo, todos los miembros pueden disponer de ella para sostenerse.

Tomando en cuenta estas consideraciones y sobre todo el conflicto agrario con Totontepec provocado porque algunos de sus pobladores invadieron terrenos de Móctum - bajo el argumento de que estaban abandonados o que esas tierras fueron tomadas a cambio del pago de deudas-, a continuación se apuntan algunas circunstancias en las que el patrimonio individual o particular es motivo de disputa al interior de la comunidad, con esto se muestra cómo disputa la gente y sobre qué.

En los casos en los que se han dado robos pequeños en la comunidad, por lo regular son negados por quien es señalado como responsable o es acusado. Para resolver el asunto o arreglarlo, la autoridad siempre solicita un testigo, aun cuando el afectado tenga la 
convicción y pruebas fundadas en hechos y momentos de que el responsable fue tal o cual persona.

Por ser un pueblo pequeño, cuando hay daños en parcelas o solares así como riñas los que están involucrados no pueden negarlo, «no se necesitan testigos», aunque la autoridad, según sea la gravedad del asunto, puede solicitar que las personas que presenciaron la pelea o los daños intervengan como testigos, para desalentar al acusado o acusados de su pretensión de recurrir al municipio.

Si la persona que agredió o que es acusada niega su culpa, a pesar de haber testigos, y «si se pone muy confiado de que en el municipio lo van a ayudar», se le multa —esto según una autoridad interna-. Si después de haber llegado a un acuerdo la persona que está obligada a pagar o a cumplir no lo hace, se le puede señalar una multa — que va al «ahorro» de la agencia — y obligarlo a pagar. El «ahorro» de la agencia es una especie de «caja chica» que se forma con las aportaciones económicas o «cooperaciones» de los miembros de la comunidad; son fondos que se utilizan para que las autoridades realicen distintas gestiones.

Todos los asuntos entre los ciudadanos — problemas o conflictos — pasan al agente, sin embargo, cuando los que están implicados se «arreglan», ya no es necesario. El agente sólo actúa cuando se presenta una queja y no se quiere reconocer el daño. De igual forma, se dieron asuntos que llegaron con la autoridad o a la agencia y que los particulares involucrados buscaban un posible arreglo o denunciaban el incumplimiento de un arreglo previo.

Cuando el comisariado no puede solucionar un problema, por ejemplo, que se cuestione la propiedad comunal de la tierra, es decir, que uno de los miembros de la comunidad u otra persona externa a ella se ostente como propietario único de todo o parte de las tierras comunales, ${ }^{12}$ este tipo de asuntos se plantean y resuelven en asamblea, como ocurre en Totontepec. En casos como éste se puede observar la influencia del Derecho positivo 
estatal, pues según el Derecho de propiedad que éste postula una persona es dueña de una cosa mueble o inmueble por usarla durante un tiempo determinado - usucapio o prescriptio, según sea el caso-, lógica o razonamiento que no impera en el derecho construido en la comunidad, ya que una persona tiene derecho de usar la tierra por ser miembro del colectivo y no al revés, es decir, usa la tierra: luego entonces es miembro. Si el problema es sobre tierra — uso y posesión — pero hubo daños, golpes o lesiones sobre las personas, se turna con el agente.

De lo anterior se puede deducir que además de las instancias formales de la comunidad, es decir, la asamblea, el agente o el comisariado de bienes comunales, existen otros ámbitos de resolución de conflictos como el que se da entre particulares o personas involucradas, así como el nivel legal de las autoridades de la cabecera municipal, como la alcaldía, que funge como juzgado. También se observa que en asuntos de menor gravedad — como el abandono de hijos, desde la perspectiva del Derecho positivo - las autoridades solo intervienen cuando así se les solicita, ya que por lo general se quedan con sus abuelos.

\section{LA PROPIEDAD COMUNAL COMO ASUNTO RELEVANTE DE LA COMUNIDAD}

El cuestionamiento al sistema de propiedad comunal se considera un atentado a la integridad de las tierras de Móctum, por ello se respeta la propiedad del producto cultivado pero no sobre la tierra. Esto en efecto ha propiciado conflictos con personas que exigen que se respete la propiedad sobre un terreno que ha cultivado o en el que tiene siembra.

En cuanto al uso de la tierra tenemos que se han establecido varios acuerdos de asamblea, por ejemplo: si una persona ya rozó - limpió y quemó- el lugar que va a sembrar, por ese hecho ya tiene derecho sobre ese pedazo de tierra, y tiene derecho a que se le respete para sembrar y cuando se cosechan los productos — maíz o frijol, principalmente-; si ya no se vuelve a rozar o a usar, otra persona puede utilizar ese pedazo de tierra. Después de usar una porción o parcela de tierra por uno o dos años, debe dejársele descansar por lo 
menos un año ya que «así se acordó por la asamblea». Si usa el mismo pedazo dos años consecutivos no puede extenderse «hacia los lados». No se permite cercar, sólo si «se trata de ganados» y siempre y cuando se «pida permiso».

De igual forma el uso de la tierra es un derecho, pero también implica una obligación, pues si una persona roza su terreno y el fuego se sale de control debe pagar los daños aunque haya sido por imprudencia y no intencional.

Cada persona tiene un solar que se le asigna por el comisariado después de cumplir algunos requisitos, como hacer servicio a la comunidad, cargos y tequios y «que se vea o exprese su voluntad» de que se va a quedar a vivir en la comunidad. En todos los casos, cada solar incluye una extensión de tierra que se puede usar para construir una casa y cultivar milpa - maíz - o frijol. A veces se dan casos en que uno de los vecinos se pasa de los límites señalados — mojoneras - y es cuando se pide la intervención del comisariado. El consejo de vigilancia se cerciora de esto trasladándose al lugar de los hechos escuchando a las partes y haciendo mediciones para enterarse de lo que sucede, es decir, hace una inspección in situ, en el lugar. En caso de que alguna de las partes en disputa haya movido las mojoneras se regresan a su lugar, y si el que las movió es renuente se le puede meter a la cárcel o bien se le puede multar por no obedecer lo que ha dispuesto la autoridad.

Otro tipo de daños al patrimonio de las personas se da cuando un ganado - chivos, mulas, burros, bueyes o vacas - entra a una milpa y no existe arreglo entre el dueño del ganado y el dueño de la milpa; el comisariado, por su parte, toma en cuenta los siguientes criterios para determinar el pago del daño: si el ganado se comió la planta pero no el fruto — la mazorca —, el dueño del animal debe pagar 50\% — porque la planta puede crecer y producir-; si el ganado se come la mazorca, el dueño del ganado debe pagar 100\%; en ambos casos el porcentaje se obtiene sobre el trabajo invertido, es decir, los días de trabajo — a \$120 por día, en el año 2006- proporcionales al daño cometido entre 10 o 20 
plantas dañadas, o bien en este último caso la entrega del número de mazorcas que el ganado o los ganados se hayan comido o dañado.

\section{DIMENSIONES DE LA NORMA NO ESCRITA}

Se puede afirmar que el proceso de gestación o génesis de una norma empieza en el momento en que un asunto es presentado a las autoridades - asamblea, agente o comisariado-, por ejemplo, solicitar un solar no implica la existencia de una parte contraria o adversario — claramente identificado-, aunque se puede presentar alguien argumentando en favor o en contra de la solicitud, revelándose disputas pasadas con el solicitante. La resolución de este tipo de conflictos o casos conlleva la aplicación de distintos criterios relacionados fundamentalmente con la circunstancia y posición de las personas ante la comunidad, y también si existe un caso similar que oriente a las autoridades y a las partes involucradas a buscar una solución. Así, son dimensiones de la norma oral la existencia de casos semejantes anteriores que se toman como referentes, y la norma como criterios surgidos a partir de la resolución de casos que no son similares pero que ayudan en la definición de esos criterios para atender «nuevos» casos. Como ya lo mencioné, uno de los derechos de los miembros de la comunidad es solicitar un solar o usar una parcela, este derecho era exclusivo de los hombres, sin embargo, se ha dado el caso de mujeres que han solicitado este derecho, lo que para las autoridades significa actuar sin casos precedentes porque las mujeres nunca lo habían solicitado.

\section{CUANDO LAS MUJERES SOLICITAN TIERRA DENTRO DE LA COMUNIDAD}

En este caso, se ilustra cómo surge la necesidad de formular una norma, las condiciones en que se aplica y la idea que se tiene de ella.

Entre las mujeres, sobre todo del grupo Mujeres Olvidadas del Rincón Mixe — grupo de mujeres formado en Móctum para promover proyectos productivos, como un molino, cultivo de hortalizas...-, se produjo un fuerte malestar o inconformidad porque decían que la asamblea de los comuneros ${ }^{13}$ acordó que las mujeres que se casaran con hombres «de fuera» tenían que abandonar Móctum. Tanto las mujeres como las autoridades 
señalan que esto fue a raíz de que en una ocasión una mujer se casó con un hombre que no era de la comunidad — que «era de fuera»— y este solicitó ser comunero; la asamblea y las autoridades lo aceptaron sin condición. Pasado el tiempo ya no quiso cumplir con los servicios, entonces le exigieron a la esposa que cumpliera en lugar de su esposo, pero ella también rehusó, por lo que «prácticamente les pidieron que se fueran».

El trasfondo de lo anterior es mantener la idea de unidad en torno a la comunidad y garantizar la protección de la integridad de la tierra como un bien económico y de pervivencia de la comunidad, así como de cada uno de sus miembros. Esto se entiende si se toma en cuenta que en la visión y versión de los habitantes de Móctum sobre el conflicto agrario fueron perdiendo sus tierras por deudas contraídas con comerciantes de Totontepec, y también porque al ser pocos habitantes los de Totontepec aprovecharon para trabajarlas y apropiarse de ellas dando algunas en arrendamiento y/o a medias.

En las entrevistas realizadas para indagar más sobre este caso, una de las autoridades agrarias señaló que las mujeres siempre han tenido derechos sobre las tierras, pero para eso tienen que cumplir obligaciones con la comunidad, sin embargo, otros reconocen que el derecho de las mujeres como comuneras es reciente. Según lo dicho por esta autoridad, ${ }^{14}$ antes no se exigían obligaciones para ser comunero o vivir en Móctum, lo único que hizo el pueblo y las autoridades es ser más estrictos, justificando esto con «el reglamento» oral o los acuerdos de la asamblea. Se puede deducir, entonces, que el reglamento sintetiza normas del Derecho positivo —importadas o introducidas por el promotor agrario como representante de la Secretaría de la Reforma Agraria, institución federal parte del gabinete del Ejecutivo Federal o Presidente- y las que resultan de su aplicación en la comunidad a casos concretos, es decir, el Derecho en la comunidad o Derecho indígena. De esta manera, la interlegalidad como fenómeno de relación entre sistemas jurídicos diversos — muchas veces influenciados e imbricados - da lugar a un sistema de normas y criterios de aplicación e interpretación propios o sui generis — resultado que se conoce como hibridación, cfr. Orantes 2010 —. El reglamento que 
mencionaba esa autoridad, como otras personas que ya han ejercido cargos, no es un texto escrito sino un referente normativo oral que comparten los vecinos de Móctum.

Por su parte, una de las mujeres del grupo Mujeres Olvidadas del Rincón Mixe argumentó que es difícil que una mujer tenga los mismos derechos que un hombre comunero: «no puede tener los mismos derechos de comunero porque las mujeres por lo general no se quedan en un lugar [en su comunidad], si se casan con hombres de fuera se tienen que ir porque el hombre es muy difícil que se quede en un lugar que no es su comunidad». A esto se agrega la regla comunitaria de que las mujeres viudas que son comuneras, al casarse nuevamente, sus derechos pasan al hombre. Sin embargo, en 2006 se dio el caso de una mujer que siendo soltera era comunera y cumplía sus obligaciones económicas, en trabajo y de tequio, al casarse solicitó se le respetara este derecho - a seguir colaborando y a mantener a su nombre un solar — para construir su casa — que se le dio cuando era soltera - ya que su esposo también es comunero — con su propio solar.

Después de una discusión en la que hubo mujeres y hombres en contra se aceptó, pero ahora ella sabe que los gastos para la familia, que formó con su marido, son dobles porque los dos deben cumplir sus obligaciones de comuneros, además de atender la casa y a su futura familia.

El caso de esta mujer de nueva cuenta nos remite al debate sobre la norma oral, ya que revela que las normas se redefinen y no son códigos estáticos sin ser ajenos a intereses y poderes locales, sino que se adecuan a las realidades y necesidades humanas de establecer un control y un orden. Finalmente esto marca una diferencia fundamental entre la norma oral y la escrita. 
INFLUENCIAS DEL DERECHO ESTATAL EN LA CONFIGURACIÓN DEL DERECHO EN LA COMUNIDAD

Para analizar la norma escrita, me baso en el Reglamento Interno introducido por la Secretaría de la Reforma Agraria, ya que es uno de los aspectos en los que se permite observar las dinámicas jurídicas de Móctum como campo semiautónomo, porque el establecimiento de cada norma revela los recursos y aspectos relevantes sobre los que la comunidad, autoridades y líderes, están preocupados de controlar. Desde la Ley Federal de Reforma Agraria de 1970, ejidos y comunidades estaban obligados a regular su funcionamiento a través de un Reglamento Interno que debía registrarse en el Registro Agrario Nacional, RAN, sin embargo, muchos núcleos agrarios no contaban con él. Dadas las modificaciones de 1992 al artículo 27 constitucional en materia de ejidos y comunidades agrarias, esa obligación no cambió, aunque la «obligatoriedad» de contar con un reglamento se hizo más imperativa a propósito del llamado Programa de Certificación de Derechos Ejidales y Titulación de Solares Urbanos, Procede, pues el reglamento de la Ley Agraria en esta materia señala como requisito indispensable que los núcleos agrarios que se integran al Programa deben contar con su Reglamento Interno además de otros documentos. ${ }^{15}$

Desde la perspectiva del Derecho positivo mexicano — según la Ley Agraria de 26 de febrero de 1992 - ${ }^{16}$ se conoce como Reglamento Interno — para el caso de los núcleos agrarios ejidales - o Estatuto Comunal — para los núcleos agrarios de bienes comunales o comunidades - al conjunto de reglas escritas que se refieren a distintos temas como: derechos de ejidatarios y/o comuneros, delimitación y aprovechamiento de las tierras, derechos de los avecindados — si los hubiera—, formas de organización, etc. Tanto uno como el otro tienen que estar registrados en el Registro Agrario Nacional, y los ejidatarios o comuneros u otras personas — según sea el caso — deben cumplirlo siempre y cuando ese Reglamento no contradiga la Ley Agraria - esto, desde la lógica jurídica del Derecho positivo mexicano, artículos 10 y 107 de la Ley Agraria. ${ }^{17}$ 
Como se había apuntado, la necesidad de contar con normas escritas ha sido parte de una exigencia del Estado, pero también las comunidades indígenas lo han retomado como una manera de defender sus derechos a tener una forma de organización interna propia y a un sistema de justicia basado en los usos y costumbres —el caso de Móctum como de otros poblados es representativo de estos procesos.

\section{DOS LÓGICAS JURÍDICAS IMBRICADAS: LA NORMA ESCRITA}

En el ejercicio de mostrar la norma escrita, se corre el riesgo de no reconocer su dinamismo, por lo que resulta necesario abordar las prácticas de justicia para observar cómo se actualizan. En el caso que ahora se aborda, se puede destacar que la simple trascripción de la norma no representa las dinámicas sociales y usos del derecho y del poder, esta descontextualización de la norma hace difícil comprender las razones y motivos involucrados en su redacción. Pese a esto, es interesante destacar que en el Estatuto Comunal de Móctum (1995) quedaron plasmadas las normas de la expulsión de comuneros y la de privación o suspensión de derechos, aun cuando éstas, en el sistema jurídico mexicano, pueden considerarse violatorias de derechos humanos, pese a que fue un agente del Estado quien estuvo presente en la redacción del documento final.

Como se ha apuntado, el Reglamento Interno o Estatuto Comunal es un ejemplo palpable de la influencia del Derecho mexicano en la dinámica interna de la comunidad y su sistema jurídico. Con la finalidad de observar esta influencia a continuación expongo a grandes rasgos el capitulado del Reglamento Interno de Móctum ${ }^{18}$ que fue elaborado con la intervención de un representante de la Promotoría Agraria de la Secretaría de la Reforma Agraria. Este documento resultó de una actualización realizada el 26 de agosto de 1995 después de que se declara finiquitado el conflicto agrario entre Móctum y Totontepec, aunque fue hasta 1999 que se consideró del todo resuelto. En esa fecha los comuneros de Móctum aprobaron la «actualización» ${ }^{19}$ de su Estatuto Comunal, ${ }^{20}$ en el cual señalaron algunos derechos y obligaciones. ${ }^{21}$ 


\section{CAPITULADO DEL ESTATUTO COMUNAL}

El Capítulo I de disposiciones generales, contiene seis artículos, de 01 a 06, de los cuales son de destacarse el 01, 03 y 06. El artículo 01 señala la necesidad de aplicar el Derecho estatal según su nivel jerárquico:

Artículo 01. En lo no previsto en este [Estatuto Comunal] se aplicará supletoriamente en la siguiente base, en su caso la carta magna, el código penal del Estado Libre y Soberano de Oaxaca, y la Ley Reglamentaria, es decir, la Nueva Legislación Agraria.

Visto desde un análisis del poder, en este artículo se muestra cómo el Derecho estatal se sobrepone y sus agentes se encargan de que impere sobre cualquier otra forma de derecho. En el artículo 03 se describe la comunidad de Móctum, sus características agrarias geográficas y categoría política, y en el artículo 06 se señala que el compromiso que contraen los comuneros es luchar por conservar su condición de bienes comunales.

\section{CAPÍTULO II DE LOS COMUNEROS}

Consta de ocho artículos, de 07 a 14, en estos se señalan algunos de los derechos de los comuneros así como sus obligaciones, por ejemplo, cómo las mujeres pueden ser comuneras, la edad máxima para ser comunero, los requisitos para ocupar «cargos mayores», la pérdida de la calidad de comunero y los casos de expulsión — si bien la Ley Agraria habla de separación.

\section{CAPÍTULO III DE LOS ÓRGANOS INTERNOS DE LA COMUNIDAD}

Se integra de 26 artículos, de 15 a 40, en los que se señalan los órganos de representación de Móctum así como sus funciones, 15 a 22 y de 32 a 34. Sin embargo, no se menciona al agente de policía, aunque en el artículo 16 se apunta que los comuneros se coordinarán con la autoridad municipal para las gestiones relacionadas con los servicios comunitarios.

Este capítulo es el más extenso de todos, pues a pesar de su título también se señalan sanciones (23 a 31). Si se asumiera una postura estrictamente legalista, según el Derecho 
estatal, hubiera sido conveniente un capítulo de sanciones. También se mencionan otros derechos de la comunidad y de los comuneros - 36, 37, 39- así como una solicitudcompromiso de la comunidad frente a la Promotoría Agraria para recibir su orientación, ${ }^{22}$ lo que muestra el afán de control del Estado a través de sus agentes.

\section{UN COMPROMISO CON LA SECRETARÍA DE LA REFORMA AGRARIA}

Ese afán de control del Estado sobre las dinámicas comunitarias es una constante que se expresa a lo largo del Estatuto. En este sentido, lo que a continuación se asienta en el Estatuto Comunal es convincente, pues acudir o no a las instancias de gobierno es una potestad o derecho y no una obligación de los núcleos agrarios, más si es sólo por asesorías.

Artículo 40. La Asamblea general de comuneros pide y manifiesta de que cuando se tenga algún caso que sea tratado en una Asamblea se pida orientación necesaria de la Coordinación de la Secretaría de la Reforma Agraria o en su caso a la Promotoría Agraria, a efecto de realizar la orientación correspondiente y sean aclaradas todas las dudas.

Es importante comentar que en la fecha que resultó este Estatuto, por ley la Secretaría de la Reforma Agraria ya no tenía obligación ni facultad de elaborar el Estatuto o prestar asesoría para ello, en todo caso le corresponde a la Procuraduría Agraria, siempre y cuando lo solicite el núcleo agrario comunidad o ejido (artículos 135 y 136 de la Ley Agraria).

Por lo demás, en este Capítulo III se señalan aspectos que la Ley Agraria marca y que lo único que se hace en el Estatuto Comunal es trascribirlos con algunas modificaciones. 
TRANSITORIOS

Son tres los artículos en este apartado y se refieren a la vigencia del Estatuto Comunal, es decir, desde qué fecha empieza a ser obligatorio o a aplicarse, así como la obligación del comisariado de hacer los trámites para que el Estatuto se inscriba en el Registro Agrario Nacional, y el «derecho irrenunciable» de la asamblea para modificarlo.

\section{DIMENSIONES DE LA NORMA ESCRITA}

La norma escrita en Móctum responde a las exigencias del Estado y su derecho que marca como necesario que esta comunidad deba ser reconocida por él como comunidad agraria. Después de la elaboración del Reglamento Interno por escrito, para integrarse a Procede la comunidad elaboró otro reglamento, ahora con la presencia de la Procuraduría Agraria, que sigue al pie de la letra lo que señala la Ley Agraria — para ser aceptado por el Registro Agrario Nacional-, por lo que no muestra aspectos relevantes para la comunidad como la expulsión. Pese a esto, los comuneros y comuneras saben que el Estatuto Comunal anterior a Procede es válido y se remiten a él cuando surgen conflictos o disputas.

El hecho de existir dos Estatutos Comunales o Reglamentos Internos por escrito, uno expuesto solo en su capitulado y elaborado con el promotor agrario justo al momento de las modificaciones al artículo 27 constitucional, el otro elaborado con la Procuraduría Agraria propiamente en el procedimiento del Procede, ambos con funciones distintas, muestra cómo la comunidad se adecua a las exigencias del Estado y construye y trasforma su propio derecho. También muestra la imbricación de sistemas y lógicas distintas, así como la subalternidad de las comunidades indígenas que son partícipes, en alguna medida, de las trasformaciones de campos sociales más amplios como el municipal. De esta manera, se tiene por un lado que para Móctum era cumplir un requisito formal que le permitió integrarse a Procede — para delimitar sus tierras solo por lo que toca a la parte de colindancias con otros núcleos agrarios- y con ello dar por concluido su conflicto agrario con Totontepec - lo que ha significado para Móctum recibir más apoyos económicos federales y municipales-; por otro, el Estatuto elaborado 
antes de Procede da a los miembros de la comunidad y a sus autoridades la seguridad de que normas como la expulsión fueron avaladas por autoridades del Estado.

Esta doble dimensión de la norma escrita como ley del Estado y como norma interna de la comunidad se puede observar cuando las autoridades resuelven disputas o conflictos e invocan la ley del Estado, como el Código Penal y la Constitución, para legitimar su actuar - aunque no citan artículos-. Por ello, la norma escrita en Móctum tiene una doble dimensión, pues no solo es el Reglamento Interno o Estatuto Comunal, como lo llama la Ley Agraria, sino también el Derecho positivo mexicano interpretado y aplicado por las autoridades según su propio entendimiento - influenciado y determinado por el contexto social y étnico-cultural en el que se desenvuelven-. Sin embargo, algunas veces esta percepción de dos formas de derecho no es tan clara, precisamente porque los sistemas jurídicos son vistos por los actores como un solo referente legal — por ejemplo en materia agraria, el criterio de dos años de abandono de la tierra por el que se considera que un comunero ya no tiene derechos, es citado por el comisariado pero también fue una norma de la Ley Federal de Reforma Agraria-, por lo que para el observador — sobre todo si no es abogado o especialista - es difícil distinguir hasta dónde se trata de una práctica comunitaria «tradicional» o de la aplicación del Derecho mexicano a las dinámicas locales, adecuaciones que pueden incluso tergiversarlo o trasgredirlo. Sin embargo, los actores y sobre todo las autoridades indígenas tienen la firme convicción de que es «su» derecho y de que se está «haciendo justicia».

\section{A MANERA DE CONCLUSIÓN}

El Reglamento Interno o Estatuto Comunal como norma escrita de Móctum no abarca todas las conductas posibles que pudieran adoptar los comuneros o comuneras de Móctum, es en todo caso la puesta en papel de las normas no escritas, lo que resultó de la participación de agentes del Estado, cuya tarea prioritaria era implementar un programa gubernamental llamado Procede. Esta actitud exhibe la intervención del Estado en la vida de las comunidades indígenas, a través de la política agraria de dotar de tierra y reconocer la que han tenido en posesión ancestralmente — los llamados procedimientos agrarios- 
lo que finalmente marca un continuo con la política colonial hacia estos pueblos.

Algunas de las normas no escritas tuvieron origen durante el conflicto con Totontepec, como el hecho de establecer la prohibición impuesta a las autoridades externas de detener a cualquier comunero de Móctum; que las mujeres que se casaran con hombres de otra comunidad se fueran — norma, que confiesan algunos que fueron autoridades, fue la «estrategia» para que los de Totontepec no quisieran retomar las tierras que Móctum había recuperado- - El cultivo de caña y la cría de ganado también entran en ese tipo de normas. Esta situación no se expone en el Estatuto Comunal (1995), por lo que es limitado frente a las normas orales o no escritas vigentes. En este sentido, la norma no escrita expresa con mayor nitidez la dinámica de la comunidad, sus asuntos y conflictos relevantes contemporáneos, así como las respuestas que la comunidad da para enfrentarlos.

Aunque se señala que el Estatuto Comunal o Reglamento Interno elaborado con la intervención del funcionario de la Promotoría Agraria era una actualización de otro existente con anterioridad, ni el agente ni el comisariado en turno lo conocían. En el Estatuto de Móctum no se anotan qué artículos se modifican y cuáles quedan sin alteración - lo que se tendría que especificar en los artículos transitorios.

Los aspectos más importantes que determinan la creación y aplicación de las normas en Móctum - y por tanto, la dinámica de su orden jurídico - están relacionados con el ámbito agrario de la comunidad: tierra, trabajo colectivo, adscripción o reconocimiento de comuneros. La confrontación de la norma escrita con las prácticas de resolución de conflictos y sus normas no escritas exhiben las limitaciones de aquélla fundamentalmente porque no muestran los casos de excepción — que son los que se dan en la cotidianidad, como fue el caso del emigrante o del comunero que incumple sus deudas económicas, que se explica con más detalle en mi trabajo doctoral (cfr. Cruz 2007)—, sino que se presentan como postulados categóricos inamovibles. Por tanto, en el caso de Móctum la exposición y puesta en marcha de su sistema jurídico — creación y aplicación de normas, 
autoridades y mecanismos de resolución de disputas, ámbitos de aplicación de la norma- no se puede reducir a lo escrito en el Estatuto Comunal o en el Reglamento Interno, en el que se pretendió por parte de los agentes del Estado trascribir, trasponer o traducir todo o parte de la Ley Agraria, eludiendo e ignorando las condiciones reales de la comunidad.

Tal situación salta a la vista cuando observamos que uno de los elementos fundamentales de la comunidad es la tierra, sin embargo, en el Estatuto no se establece nada más que generalidades como ámbito territorial de la jurisdicción de las autoridades y respecto a su posesión y tipo de usufructo, pese al proceso histórico de reivindicación y de que los conflictos más relevantes están estrechamente relacionados con ella y con la aceptación de nuevos comuneros — cumplimiento de obligaciones y ejercicio de derechos.

También se podrá observar que los acuerdos actualizan la norma escrita. De esta manera, una norma no solo es un breve enunciado de una conducta sino la regla que resulta de tomar en cuenta un hecho y sus circunstancias, y al individuo en su relación con la comunidad, por ello, la actualización de la norma se da a través de la exposición del asunto ante la asamblea, o bien cuando el agente «hace justicia». Así, tanto las normas escritas como las no escritas están rodeadas de situaciones y antecedentes que les dan sentido, lo cual está en la memoria de las autoridades dando legitimidad a un acuerdo de que tal norma debe ser aplicada.

Las limitaciones de la norma escrita, es decir, el Reglamento Interno, se deben en parte a lo antes apuntado, pero también a la intervención de la Promotoría de la SRA, ya que su interés era más el cumplir un programa de gobierno que el apoyar a Móctum, por ejemplo, a recuperar y sistematizar su historia y su sistema jurídico. Esto es evidente cuando en un asunto la autoridad se refiere al «Reglamento» más como el conjunto de acuerdos y precedentes fácticos y prácticos que a la norma escrita. Si los de Móctum aceptaron hacer el Reglamento Interno fue porque les convenía incorporarse a Procede — aun cuando son comunidad y no ejido - por el conflicto agrario con Totontepec, pues 
el deslinde, ubicación de mojoneras o marcas de límites, así como la delimitación de sus tierras para tener «seguridad jurídica» debió hacerse desde 1975; sin embargo, lo lograrían con este procedimiento en los umbrales del siglo XXI. El finiquito del conflicto agrario marcó la posibilidad de Móctum de establecer otro tipo de relación con Totontepec, lo que se expresa en la «cooperación» entre autoridades — que se desarrolla a detalle en la tesis-, que observamos recurrentemente como en el caso de la expulsión y también en la inyección de recursos municipales para obras de infraestructura comunitaria, como la electrificación trifásica y el entubado del agua potable.

Finalmente, el control del Estado es cada vez mayor sobre las formas comunitarias de «hacer justicia», debido entre otros aspectos al desarrollo de los medios de trasporte y comunicación. De esta manera, las autoridades de Móctum no son ajenas a la intervención de los agentes del Estado mexicano como la Procuraduría de Justicia del Estado, la Procuraduría Agraria y, sobre todo, la Comisión de Derechos Humanos de Oaxaca. En esta interacción se da una lucha de poder entre sistemas jurídicos en que los protagonistas - las autoridades indígenas, las no indígenas y los usuarios en las comunidades - tratan de hacer que uno impere sobre el otro, o bien que subsista reforzando la práctica comunitaria del trabajo colectivo y de los cargos, así como en la reafirmación del comunitarismo basado en una ideología de la «armonía». Dicho discurso comunitarista puede ser usado con el fin de promover la solidaridad local convirtiéndose en una estrategia para resistir la hegemonía del Estado, buscando reinstalar el balance entre las partes en disputa. Esto puede verse como la intención de establecer el equilibrio de las relaciones sociales que se había visto alterado por el conflicto.

De esta manera, los valores culturales que predominan en el proceso de impartición de justicia son los de ayuda mutua, balance, armonía e igualdad. Con todo ello, se pretende inhibir tanto la intervención de agentes externos como los intentos de los habitantes de la comunidad para acudir a la «justicia del Estado», y que eludan sus obligaciones ante el colectivo, o para «realmente» defenderse. Pero también es importante advertir que en el ejercicio de «hacer justicia» intervienen otros factores que pueden suponer un abuso de 
las autoridades indígenas y del colectivo comunitario sobre el individuo; sin embargo, no se puede afirmar esto de manera categórica y generalizada sin hacer un conocimiento y estudio profundo de los casos — sus circunstancias y usos del poder que los enmarcanasí como el contexto comunitario en el que se dan y con los que se pretende ejemplificar y respaldar tal afirmación.

Foto 1. Móctum visto desde el panteón comunitario. Al fondo Totontepec Villa de Morelos

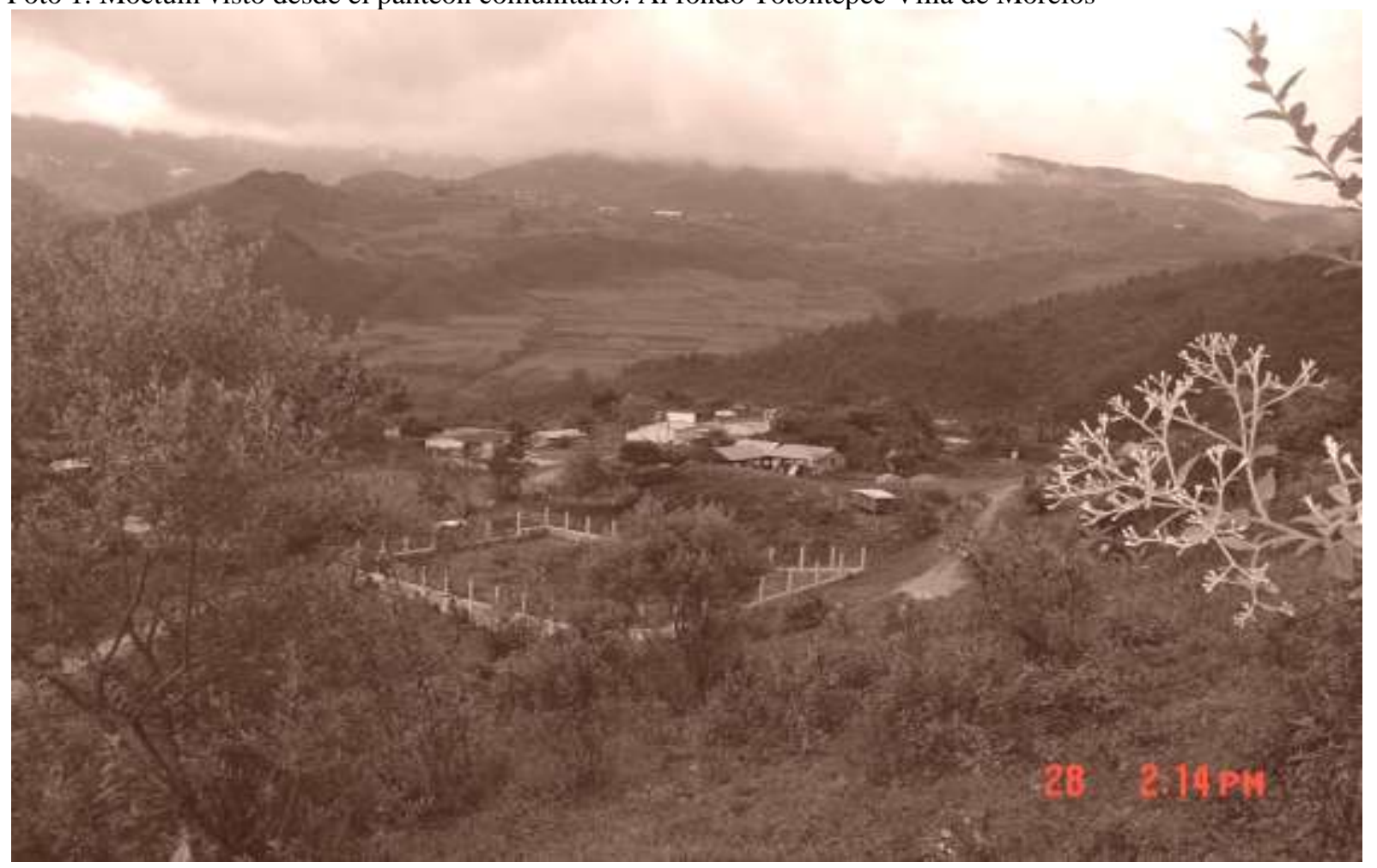

Fuente: Cruz Rueda, Elisa, 2004. 


\section{BIBLIOGRAFÍA}

Congreso de los Estados Unidos Mexicanos, 1992, «Ley Agraria», Orden Jurídico Mexicano, en http://www.ordenjuridico.gob.mx/Federal/Combo/L-2.pdf [consulta: 3 de abril de 2010].

Cruz, Rueda, Elisa, 2004, «Normas y procesos en San Marcos Móctum: expresiones del derecho mixe», en Haciendo justicia. Interlegalidad, derecho y género en regiones indígenas, editado por Sierra Camacho, María Teresa, Cámara de Diputados LIX Legislatura/Centro de Estudios Superiores en Antropología Social/Miguel Ángel Porrúa, México, pp. 299-357.

2005, «Los principios generales del derecho en Totontepec Mixe, Oaxaca», ponencia presentada en el Encuentro Pueblos y Fronteras, Pre-Congreso de la Red Latinoamericana de Antropología Jurídica, San Cristóbal de las Casa, Chiapas, 5-9 de octubre de 2005, Programa de Investigaciones Multidisciplinarias de Mesoamérica y el Sureste/Universidad Autónoma de Chiapas.

2007, «Dinámicas jurídicas, construcción del derecho y procesos de disputa en una comunidad indígena de Oaxaca», Tesis de doctorado, Universidad Autónoma Metropolitana Unidad Iztapalapa, México.

2008, «El sistema jurídico en Totontepec Villa de Morelos: como campo jurídico y como nivel legal», en La Defensa de los Derechos de los Pueblos Originarios, Afroamericanos y Migrantes. XVI Jornadas Lascasianas Internacionales, coordinado por José Emilio Rolando Ordóñez Cifuentes, Instituto de Investigaciones Jurídicas-UNAM, México, pp. 143-165.

Gómez Bravo, Noemí, 2004, Móctum: Antigua grandeza de un pueblo mixe, Fondo para la Cultura y las Artes/Gobierno del estado de Oaxaca, Oaxaca. 
Krotz, Esteban, 1995, «Órdenes jurídicos, antropología del derecho, utopía. Elementos para el estudio antropológico de lo jurídico», en Pueblos Indígenas ante el derecho, coordinado por Chenaut, Victoria, María Teresa Sierra Camacho, Centro de Investigaciones y Estudios Superiores en Antropología Social/Centro Francés de Estudios Mexicanos y Centroamericanos, México, pp. 345-353.

Martínez Luna, Jaime, 2003, Comunalidad y Desarrollo, Centro de Apoyo al Movimiento Popular Oaxaqueño, A. C., Oaxaca.

Nader, Laura, 1998, Ideología armónica: justicia y control en un pueblo de la montaña zapoteca, Instituto Oaxaqueño de las Culturas/Centro de Investigaciones y Estudios Superiores en Antropología Social del Istmo, Oaxaca.

Orantes, José Rubén, 2010, «Nuevas Estrategias Jurídicas: Hibridación de los Derechos Mexicano e Indígena entre los Tseltales de Tenejapa, Chiapas», Protocolo de Tesis, El Colegio de la Frontera Sur, Chiapas.

Regino Montes, Adelfo, 1996, «Autonomía y derecho indígena», en Coloquio sobre derechos indígenas, editado por el Instituto Oaxaqueño de las Culturas, Instituto Oaxaqueño de las Culturas, Oaxaca, pp. 130-173.

2002, «La comunalidad. Raíz, pensamiento, acción y horizonte de los pueblos indígenas», México indígena (nueva época), v. 1, n. 2, pp. 7-14.

Santos, Boaventura de Sousa, 1991, Estado, Derecho y Luchas Sociales, Instituto Latinoamericano de Servicios Legales Alternativos, Bogotá.

1995. Toward a New Common Sense: Law, Science and Politics in the Paradigmatic Transition, Routledge, Nueva York/Londres. 
1998, La globalización del derecho: los nuevos caminos de la regulación y la emancipación, ILSA/Universidad Nacional de Colombia, Bogotá.

Sierra Camacho, María Teresa, 1987, El ejercicio discursivo de la autoridad en asambleas comunales. Metodología y análisis del discurso oral, Cuadernos de la Casa Chata, México.

Sierra Camacho, María Teresa (ed.), 2004a, Haciendo justicia. Interlegalidad, derecho y género en regiones indígenas, Cámara de Diputados LIX Legislatura/Centro de Investigaciones y Estudios Superiores en Antropología Social/Miguel Ángel Porrúa, México.

2004b, «Introducción. Hacia una interpretación comprensiva de la relación entre justicia, derecho y género: los procesos interlegales en regiones indígenas», en Sierra Camacho, María Teresa (ed.), Haciendo justicia. Interlegalidad, derecho y género en regiones indígenas, Cámara de Diputados LIX Legislatura/Centro de Investigaciones y Estudios Superiores en Antropología Social/Miguel Ángel Porrúa, México, pp. 11-56.

2006, «La renovación de la justicia indígena en tiempos de derechos: etnicidad, género y diversidad», en Estado, Violencia, Ciudadanía en América Latina, compilado por Stanley, Ruth, Vervuert, Frankfurt/Madrid.

Servicios del Pueblo Mixe SER, 2008, «Mapa: El territorio (región) Mixe», Red Indígena, en http://www.redindigena.net/ser/pueblomixe/localizacion.html [consulta: 22 de enero de 2008].

Swartz, Marc, Victor, W. Turner y Arthur Tuden, 1994, «Antropología política: una introducción», Alteridades, año 4, n. 8, pp. 101-126. 
Valdivia Dounce, María Teresa, 2001, «En torno al sistema jurídico indígena», Anales de Antropología, n. 35, Instituto de Investigaciones Antropológicas, UNAM, pp. 63-77.

2002, «Normas jurídicas y sistemas de cargos mixes», Anales de Antropología, Instituto de Investigaciones Antropológicas, UNAM, n. 36, pp. 93-116.

2010, Pueblos mixes: sistemas jurídicos, competencias y normas, Instituto de Investigaciones Antropológicas, UNAM, México D. F. 
Notas

\begin{abstract}
${ }^{1}$ Parte de los datos presentados en este trabajo son el resultado de mi tesis de doctorado (Cruz 2007) y de otros trabajos (Cruz 2004). Agradezco a Conacyt por el apoyo que me brindó durante mis estudios de doctorado así como para la realización de trabajo de campo y participación en el proyecto Conacyt U51240-S «Globalización, derechos indígenas y justicia desde una perspectiva de género y poder: una propuesta comparativa», coordinado por las doctoras María Teresa Sierra Camacho, Rachel Sieder y Rosalva Aida Hernández.

${ }^{2}$ Licenciada en Derecho por la UNAM; Mtra. y Dra. en Ciencias Antropológicas por la UAM-I. Profesora de Tiempo Completo de la Licenciatura en Gestión y Autodesarrollo Indígena de la Universidad Autónoma de Chiapas, Campus III, San Cristóbal de Las Casas. Miembro del Cuerpo Académico «Etnia, Estado y Desarrollo» e investigadora del Sistema Nacional de Investigadores como candidata.

${ }^{3}$ Sobre el debate del pluralismo jurídico y la explicación de la interlegalidad, así como sobre los datos de creación y composición municipal de este Distrito, la diversidad cultural al interior del grupo etnolingüisstico mixe, abundo en mi tesis de doctorado (Cruz 2007). De igual forma se puede consultar (Cruz 2004, Cruz 2008).

${ }^{4}$ El campo jurídico que se estudia, en su dimensión territorial se ubica en la región mixe, Sierra Norte, del estado de Oaxaca, concretamente se trata del municipio de Totontepec. Es importante advertir que el nivel federal lo enuncio en mi tesis de doctorado como parte del contexto y que únicamente me aboco al campo jurídico en los niveles estatal, municipal-regional y de la agencia. Indudablemente lo federal es importante porque enmarca la conflictividad de la tenencia de la tierra y de la relación de las cabeceras municipales y sus agencias.
\end{abstract}

${ }^{5} \mathrm{Cfr}$. http://www.redindigena.net/ser/pueblomixe/localizacion.html. Es importante aclarar que en este mapa aparece Totontepec como parte de la zona media, sin embargo los propios habitantes de la zona se identifican con la zona alta, además de que en entrevista con maestros indígenas señalan que la variante dialectal de Totontepec es más apegada a la zona alta que a la media. Por esta circunstancia en este trabajo ubico mi trabajo en la zona alta.

${ }^{6}$ En otro trabajo (Cruz 2004) hago un análisis del Derecho en Móctum y cómo las mujeres se constituyen en un elemento esencial de contestación y trasformación del mismo.

${ }^{7}$ En los asuntos que se presentaron en la agencia de policía, las audiencias eran en idioma mixe y las autoridades siempre señalaban la importancia del cumplimiento de acuerdos y de la voluntad del pueblo, pero también invocaban la Constitución y la Ley Agraria. Sin embargo, estos ámbitos del Derecho en San Marcos Móctum no son los únicos, pues se dan otros a nivel de los particulares, como los consejos que dan las personas mayores - que han pasado por todos los cargos- cuando se los solicitan, o los acuerdos entre los particulares sin llegar con la autoridad, así como los compromisos de ayuda mutua como la mano vuelta y el tequio, aspectos que Adelfo Regino (1996: 152) señala como elementos de lo que llama Derecho Mixe. ${ }^{8}$ El trabajo de Nader nos ayuda a constatar cómo los indígenas desde sus comunidades conciben sus sistemas normativos, cómo los justifican, sobre qué lógica o argumentos se erigen. El planteamiento de la comunalidad coincide con la propuesta de Laura Nader en torno a la «ideología de la armonía» cuando se refiere al discurso usado por las autoridades indígenas para mantener un cierto control sobre sus asuntos internos, evitando al mismo tiempo la intromisión del Estado. Nader documenta, de esta manera, cómo las comunidades zapotecas han buscado mantener el control social y cultural de las dinámicas internas del pueblo, entre ellas la justicia, a través de una ideología unificante que promueve la legitimidad del actuar de las autoridades y la lealtad de los vecinos. Esto es claro en el caso de San Marcos Móctum, cuando el apoyo al acusado en un futuro próximo, así como su estadía en la comunidad, se condicionan a cambio de que acepte su error, respete a la autoridad y los acuerdos de la asamblea. También la imagen de unidad que se quiere dar hacia afuera a personas ajenas a la comunidad se expresa, por ejemplo, cuando se reconocen propiedades particulares pero al exterior se dice: «la tierra es comunal» $\mathrm{y}$ «es de todos»

${ }^{9}$ Sin duda los conflictos entre católicos y adventistas son relevantes, pero no los pude documentar. En mis últimas visitas las autoridades cuidaban mucho de que se expusieran o se tratara públicamente los asuntos que les llegaban a la agencia de policía; supe que una vez que yo abandonaba la comunidad hacían asambleas, citaban a las personas involucradas en un asunto o las encarcelaban. Tal vez consideraban que mi presencia, por ser abogada, les causaba problemas en el manejo del conflicto, dato que no he 
corroborado. Así me enteré de varios asuntos que no pude presenciar, pero que la disputa se daba entre católicos y protestantes por temas que aparentemente no tenían que ver con lo religioso.

${ }^{10}$ Debido a la falta de alumbrado público, en la noche no se distingue quién anda caminando por la comunidad, ya que se han dado robos, para evitarlos todos saben que a esa hora no deben estar fuera de sus casas, por lo que si alguien es visto a esa hora es perseguido por los topiles para ser llevado a la agencia municipal. También en caso de estar ocasionando disturbios, por borrachera u otras circunstancias.

${ }^{11}$ La comunidad como grupo humano, como forma de vida colectiva y como lugar de origen y de destino, es el centro de la vida, cotidianidad y acción humana, y de la dinámica de los indígenas y sus pueblos. De esta manera, el conjunto de argumentaciones, sobre su importancia como centro que rige la acción humana y los recursos, es lo que en Oaxaca se ha llamado comunalidad (Regino 2002; Martínez Luna 2003), que más que un concepto es una forma de pensamiento y una actitud en torno a la comunidad. En este sentido, la comunalidad es a la vez el principio de unidad básica y central de cualquier conducta, proyecto, meta o fin en torno a la comunidad, es decir, es «la actitud humana hacia lo común» (Regino 2002: 7).

${ }^{12}$ En mi tesis de doctorado (Cruz 2007), expongo este y otros casos dados tanto en Móctum como en Totontepec.

13 Antes de 1999 la Asamblea se componía mayoritariamente de hombres, porque las mujeres viudas heredaban el derecho del marido difunto pero no se atrevían a opinar, ahora las mujeres jóvenes pueden ser comuneras si lo solicitan y cumplen proporcionalmente como los hombres con los tequios y cuotas, esto gracias al trabajo de las mujeres del grupo con el apoyo de Servicios de Apoyo Intercultural, A. C., SAIAC. ${ }^{14}$ Sobre el caso que sucedió con el hombre que no quería hacer servicio la autoridad señaló: «nadie lo obligó a irse, él se fue primero y su esposa después, y según el Reglamento como ya se fueron por más de dos años ya perdieron sus derechos, si quieren regresar tienen que cumplir con los requisitos». Esto dicho por el comisariado parece ser un argumento que se opone a la idea de que en realidad hubo una expulsión.

${ }^{15}$ Sin embargo, podría entenderse que el requisito de tener un Reglamento Interno/Estatuto Comunal es más una opción que una obligación, pues en caso de no existir se aplicará la Ley Agraria, es decir, cuando surja algún problema o asunto al interior del núcleo agrario, y a falta de Reglamento o Estatuto, la autoridad interna o representante se auxiliará o aplicará la Ley Agraria o sus usos y costumbres. Cuando el asunto o problema llegue a los Tribunales Agrarios y a falta de Reglamento — ejidos- o Estatuto - comunidades - , los jueces atenderán lo que diga la Ley Agraria, y en caso de comunidades indígenas además sus usos y costumbres. Pese a lo anterior, la Ley Agraria menciona aspectos que no regula, para ello remite al Reglamento o Estatuto, en donde deben regularse por la comunidad de acuerdo con sus circunstancias.

${ }^{16}$ Diario Oficial de la Federación (DOF) de 26 de febrero de 1992.

17 «Artículo 10. Los ejidos operan de acuerdo con su reglamento interno, sin más limitaciones en sus actividades que las que dispone la ley. Su reglamento se inscribirá en el Registro Agrario Nacional, y deberá contener las bases generales para la organización económica y social del ejido que se adopten libremente, los requisitos para admitir nuevos ejidatarios, las reglas para el aprovechamiento de las tierras de uso común, así como las demás disposiciones que conforme a esta ley deban ser incluidas en el reglamento y las demás que cada ejido considere pertinentes. ... Artículo 107. Son aplicables a las comunidades todas las disposiciones que para los ejidos prevé esta ley, en lo que no contravengan lo dispuesto en este Capítulo [De las comunidades, artículos 98 a 107]. ... Artículo 99. Los efectos jurídicos del reconocimiento de la comunidad son: IV. Los derechos y las obligaciones de los comuneros conforme a la ley y el estatuto comunal».

${ }^{18}$ El contenido de cada artículo se desarrolla en la tesis de doctorado (Cruz 2007),

${ }^{19}$ Primera hoja supra, parte inicial. Aunque se dice actualización, nadie de los que fueron y son autoridades pudo darme razón de un anterior Estatuto. El párrafo introductorio del estatuto comunal señala: ACTUALIZACIÓN DEL ESTATUTO COMUNAL DEL POBLADO DENOMINADO SAN MARCOS MÓCTUM, MUNICIPIO DE Totontepec VILLA DE MORELOS, DISTRITO DE SANTIAGO Zacatepec MIXE, ESTADO DE OAXACA, EN ASAMBLEA GENERAL DE COMUNEROS CELEBRADA POR PRIMERA CONVOCATORIA DEL DÍA 26 DE AGOSTO DE 1995.

${ }^{20} \mathrm{El}$ estatuto comunal es un documento elaborado por los comuneros y pobladores en general, está bajo resguardo del Agente de Policía. Este documento cuenta de cinco páginas tamaño oficio, cada una de ellas 
presenta cuatro sellos: «Comisariado de Bienes Comunales», «Consejo de Vigilancia» y «Agencia de policía» de San Marcos Móctum, y de la Secretaría de la Reforma Agraria (SRA) «Promotoría Agraria, Mixe, Oax.» — no tiene número de folio o de registro en el Registro Agrario Nacional一. Se compone de cuarenta artículos numerados con números arábigos y tres capítulos titulados y señalados con números romanos, así como una parte de artículos transitorios marcados con números ordinarios en letras mayúsculas.

${ }^{21}$ Es importante precisar que existe otro Estatuto Comunal aprobado por la asamblea de comuneros de Móctum, de 9 de septiembre de 1998, sin embargo, cuando yo empecé a investigar el Estatuto, las autoridades no tenían copia y sólo me entregaron el documento que es objeto de análisis en la tesis. Sabía que con Procede el Estatuto debía inscribirse en el Registro Agrario Nacional, RAN, por lo que mi intención fue cotejar el que yo tenía y el que quedó inscrito para saber si era el mismo. Sin embargo, desde enero de 1999 lo solicité a la delegación de RAN en Oaxaca y me dieron largas. En septiembre de 2003, y a duras penas - pagando los respectivos derechos en el banco, y esperando varios meses- conseguí copia certificada de la carpeta básica, así como del Estatuto Comunal registrado.

${ }^{22}$ En 1992 entra en vigor la nueva Ley Agraria, en la cual se establecía la creación de la Procuraduría Agraria, a la cual, en lugar de SRA, le toca asesorar y orientar a los sujetos agrarios o campesinos. Así cuando se elabora este Estatuto Comunal ya existía PA y SRA se estaba descentralizando, incluso se impulsó el retiro voluntario de sus funcionarios, por lo que desde otro punto de vista, muchos trabajadores - sobre todo los de la estructura territorial o de campo- se dedicaron a tratar de asegurar su trabajo, o bien a aprovechar todos los medios para que los campesinos los siguieran requiriendo.

Fecha de recepción: 10 de diciembre de 2009.

Fecha de aceptación: 26 de julio de 2010. 